\title{
Aspectos destacados de las Guías de la Sociedad Europea de Cardiología sobre el manejo de adultos con cardiopatías congénitas de 2020
}

\author{
Dres. Guillermo Agorrody ${ }^{1,2}$, Lorena Dieguez ${ }^{1}$
}

\section{Introducción}

La nueva Guía europea del manejo de pacientes adultos con cardiopatías congénitas (CC) se publica diez años más tarde que su predecesora. En esta versión revisada, se enfatiza sobre la necesidad de un cuidado de por vida de los pacientes con CC, ya que se trata de una enfermedad crónica. Además, se da gran importancia al proceso que el paciente debe transitar desde los cuidados pediátricos hacia el equipo especialista en $\mathrm{CC}$ del adulto. Este equipo debe ser multidisciplinario, tomando en cuenta que el paciente, además de poseer su cardiopatía de base, puede presentar complicaciones relacionadas a las correcciones realizadas, así como enfermedades relacionadas con el envejecimiento. En este sentido, se promueve, también, la transición desde el cuidado de sus padres o tutores hacia la asunción de un rol protagónico del paciente en lo que respecta al cuidado de su propia salud.

En las actuales directrices se hacen recomendaciones generales y luego se dividen en secciones específicas de acuerdo con la cardiopatía subyacente. Se abandona el término GUCH (abreviatura de Grown Up Congenital Heart Disease) para adoptar el término ACHD (Adult Congenital Heart Disease), ya que se incluye a todos los pacientes a lo largo de su vida adulta con problemas congénitos cardíacos y se los separa de su contraparte pediátrica.

En esta revisión nos centraremos en el manejo de las principales lesiones congénitas cardíacas encontradas en la práctica clínica habitual del cardiólogo de adultos.

\section{Aspectos generales}

En la actualidad, la prevalencia de CC a nivel mundial es aproximadamente 9 cada 1.000 recién nacidos vivos. Si bien se encuentra en descenso en países occidentales con considerable avance tecnológico debido al screening prenatal, la prevalencia global de los defectos cardíacos complejos está en aumento. Esto puede ser explicado en parte por los grandes avances a nivel médico, quirúrgico e intervencionista en las últimas décadas, que han permitido que más del $90 \%$ de los pacientes con CC sobrevivan hacia la vida adulta. Hoy en día la población de adultos con CC supera ampliamente a su contraparte pediátrica. Las CC se pueden dividir en leves, moderadas o severas, según su complejidad (tabla 1), que a su vez determinará su tratamiento, pronóstico y seguimiento.

\section{Lesiones específicas}

\section{Shunts pretricuspídeos}

Dentro de este grupo se encuentran incluidos todos los tipos de comunicación interauricular (CIA) y de retornos venosos pulmonares anómalos.

Las CIA simples suelen generar shunts de izquierda a derecha, en especial si su tamaño es mayor o igual a $10 \mathrm{~mm}$, lo que causa sobrecarga de volumen del ventrículo derecho (VD) e hiperflujo pulmonar. Con el tiempo, cualquier condición vinculada al envejecimiento que reduzca la compliance del ventrículo izquierdo (VI) o de la aurícula izquierda (AI) puede aumentar el grado de shunt a través del defecto, lo que hace que esta se vuelva hemodinámicamente significativa con la edad.

1. Unidad de Cardiopatías Congénitas, Centro Cardiológico Americano, Montevideo, Uruguay.

2. Residente de Cardiología, Instituto Nacional de Cirugía Cardíaca (INCC), Montevideo, Uruguay.

Correspondencia: Guillermo Agorrody. Correo electrónico: guillermo.agorrody@gmail.com

Declaración de conflicto de intereses: Ninguno.

Recibido Jun 11, 2021; aceptado Jul 1, 2021 
Tabla 1. Clasificación de complejidad de las diferentes cardiopatías congénitas.

Leve

- Válvula aórtica bicúspide aislada.

- Estenosis pulmonar leve.

- CIA, CIV o DAP pequeño.

- CIA tipo ostium secundum, CIV o DAP reparados, sin secuelas.

\section{Moderado}

- Retorno venoso pulmonar anómalo parcial o total.

- Coronaria anómala con origen en arteria pulmonar.

- Defecto del septo atrioventricular (tipo canal AV), incluyendo CIA tipo OP.

- Coartación de aorta.

- Anomalía de Ebstein.

- DAP mediano o grande sin reparar.

- Estenosis pulmonar moderada o severa.

- CIA tipo seno venoso.

- Tetralogía de Fallot reparada.

- TGA reparada con cirugía de switch arterial.

\section{Severo}

- Cualquier cardiopatía congénita asociada a hipertensión arterial pulmonar (incluido el síndrome de Eisenmenger).

- Cardiopatía congénita cianótica.

- Circulación de Fontan.

- Atresia pulmonar.

- TGA con cirugía de Senning o no reparada.

- Truncus arterioso.

CIA: comunicación interauricular; CIV: comunicación interventricular; DAP: ductus arterioso persistente; AV: aurículoventricular; OP: ostium primum; TGA: transposición de grandes arterias.

Con respecto al tratamiento de la CIA, se abordará la del tipo ostium secundum porque es la que el cardiólogo de adultos se encuentra con más frecuencia en su práctica diaria.

La decisión de cerrar el defecto en pacientes con presiones de llenado elevadas debe incluir su oclusión transitoria con balón, para valorar el comportamiento diastólico del VI.

Por otro lado, para los pacientes con hallazgos no invasivos sugestivos de hipertensión pulmonar (HTP), se recomienda un cateterismo cardíaco derecho completo con estimación de las resistencias vasculares pulmonares (RVP), el cociente de flujos pulmonar y sistémico $(\mathrm{Qp} / \mathrm{Qs})$ y las presiones pulmonares. Se establecen unas RVP $<5$ unidades Wood (UW) con Qp/Qs >1,5 como límite superior de HTP en las CIA que permiten el cierre del defecto (tabla 2). Se contraindica el cierre cuando las RVP permanecen $\geq 5 \mathrm{UW}$, aun en presencia de tratamiento vasodilatador pulmonar específico.

Se introduce el concepto de "tratar y reparar" en los casos de resistencias elevadas ( $\geq 5 \mathrm{UW}$ ), en los que, si descienden por debajo del valor límite, puede considerarse el cierre con dispositivos fenestrados (recomendación IIb, tabla 2). Esta última estrategia requiere una valoración individualizada del paciente, teniendo en cuenta el muy mal pronóstico que presenta la HTP postcierre del defecto, que puede aparecer años después de este.

Seguimiento. Los pacientes reparados antes de los 25 años sin secuelas residuales (HTP, insuficiencia valvular, shunt residual) no requieren seguimiento periódico. De todas maneras, deben estar informados acerca del riesgo mayor de presentar taquiarritmias supraventriculares a lo largo de su vida.

Los pacientes con secuelas residuales o aquellos reparados luego de los 40 años, deben ser evaluados con regularidad. Los primeros dos años con mayor asiduidad y si no presentan complicaciones en este período de tiempo, pueden continuar con controles cada 3 a 5 años. Es fundamental realizar screening de taquiarritmias supraventriculares 
Tabla 2. Recomendaciones para el cierre de defectos septales auriculares.

Recomendaciones para el cierre de comunicación interauricular
Se recomienda el cierre de la CIA independientemente de la presencia de
síntomas, en pacientes con sobrecarga de volumen del VD sin evidencia
de DVI o HTP (descartada mediante pruebas no invasivas o cateterismo
cardíaco con RVP $<3$ UW).

Se recomienda el cierre de la CIA tipo OS en forma percutánea como técnica de elección, siempre y cuando sea técnicamente factible.

En pacientes añosos, no candidatos al cierre percutáneo, se recomienda una valoración cuidadosa de riesgos quirúrgicos vs. el potencial beneficio clínico del cierre.

En pacientes con signos indirectos de presiones pulmonares elevadas, se impone la evaluación invasiva de las RVP.

En pacientes con disfunción del VI, se recomienda realizar el test de oclusión con balón para evaluar adecuadamente el beneficio del cierre de la CIA contra el potencial impacto negativo que tendría el aumento de las presiones de llenado del VI en el pronóstico.

En pacientes con sospecha de embolias paradojales (luego de excluidas otras causas), que no presenten HTP o disfunción del VI, se debe considerar el cierre de la CIA, independientemente de su tamaño.

En pacientes que hayan desarrollado HTP con RVP entre 3 y 5 UW, debe considerarse el cierre cuando exista un shunt izquierda-derecha significativo (Qp/Qs $>1,5)$.

En pacientes que hayan desarrollado HTP con RVP $\geq 5$, podría considerarse el cierre con dispositivos fenestrados luego de objetivada la caída de las RVP con vasodilatadores pulmonares en presencia de un shunt izquierda-derecha significativo $(\mathrm{Qp} / \mathrm{Qs}>1,5)$.

No se recomienda el cierre en pacientes con fisiología de Eisenmenger, HTP con RVP $\geq 5$, a pesar del tratamiento con vasodilatadores pulmonares o desaturación durante el ejercicio (Sat $\left.\mathrm{O}_{2}<90 \%\right)$.

\section{Clase de \\ recomendación}

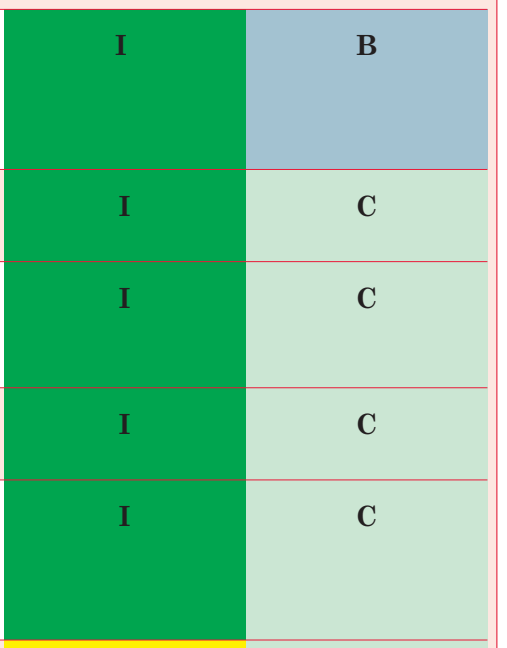

IIa

C

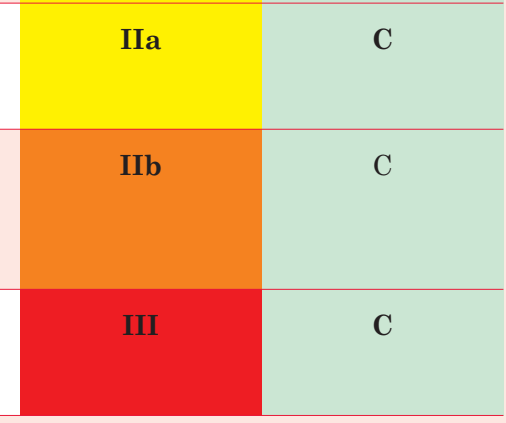

CIA: comunicación interauricular; VD: ventrículo derecho; DVI: disfunción ventricular izquierda; HTP: hipertensión pulmonar; RVP: resistencias vasculares pulmonares; OS: ostium secundum; VI: ventrículo izquierdo; Qp/Qs: relación de flujo sanguíneo pulmonar y sistémico; Sat $\mathrm{O}_{2}$ : saturación de oxígeno.

(particularmente fibrilación auricular [FA]) y de HTP postcierre en dichos pacientes.

\section{Shunts postricuspídeos}

Dentro de los shunts postricuspídeos se destacarán aspectos del manejo de comunicación interventricular y ductus arterial permeable. En este caso, la evaluación de la HTP es diferente, ya que admite niveles más altos para su cierre. Se permite su cierre con $\mathrm{RVP}<5 \mathrm{UW}$, siempre que mantenga un $\mathrm{Qp} / \mathrm{Qs}>1,5$. Este escenario particular es muy poco frecuente en los adultos y requiere de su manejo en centros con experiencia en el tratamiento de estos defectos. En estos pacientes no existe evidencia sobre la estrategia de "tratar y reparar".

El algoritmo de indicación de cierre de estos defectos (tablas 3 y 4) comienza con la presencia de sobrecarga de volumen del VI, si bien no existe un pun- to de corte establecido formal que habilite la indicación. Cabe destacar que no se incluyen RVP que contraindiquen el cierre de los defectos ni se incluye el cociente de RVP y resistencias sistémicas, que resulta de vital importancia en el algoritmo de manejo de estas patologías en la población pediátrica.

\section{Lesiones obstructivas del corazón izquierdo}

\section{Estenosis valvular, subvalvular y supravalvular aórtica}

La causa congénita más frecuente de estenosis valvular aórtica es la válvula aórtica bicúspide (BAV). Esta entidad, a su vez, se asociará en más del $80 \%$ de los pacientes con algún grado de dilatación de la aorta ascendente, lo que obliga a estudiar minucio- 
Tabla 3. Recomendaciones para el cierre de defectos septales ventriculares.

Recomendaciones para el cierre de comunicación interventricular
Se recomienda el cierre de la CIV, independientemente de la presencia de
síntomas, en pacientes con sobrecarga de volumen del VI sin evidencia de
HTP (descartada mediante pruebas no invasivas o cateterismo cardíaco
con RVP <3 UW).

En pacientes con antecedentes de EI, el cierre de CIV debe considerarse a pesar de no presentar un shunt izquierda-derecha significativo.

Debe considerarse el cierre quirúrgico de la CIV en pacientes que asocien IAo progresiva por prolapso de velo valvular aórtico.

En pacientes que hayan desarrollado HTP con RVP entre 3 y 5 UW, debe considerarse el cierre cuando exista un shunt izquierda-derecha significativo $(\mathrm{Qp} / \mathrm{Qs}>1,5)$.

En pacientes que hayan desarrollado HTP con $R V P \geq 5$, podría considerarse el cierre en presencia de un shunt izquierda-derecha significativo $(\mathrm{Qp} / \mathrm{Qs}>1,5)$, luego de una evaluación individualizada realizada en centros de experiencia.

No se recomienda el cierre en pacientes con fisiología de Eisenmenger, HTP con RVP $\geq 5$ o desaturación durante el ejercicio (Sat $\mathrm{O}_{2}<90 \%$ ).

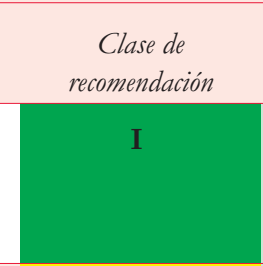

IIa C
C

IIa $\quad$ C

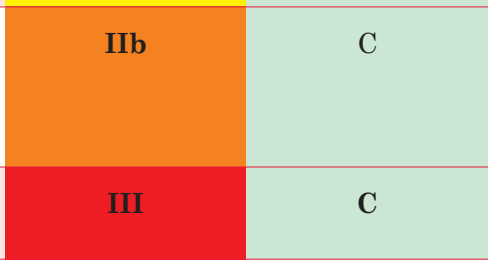

CIV: comunicación interventricular; VI: ventrículo izquierdo; HTP: hipertensión pulmonar; RVP: resistencias vasculares pulmonares; EI: endocarditis infecciosa; IAo: insuficiencia aórtica; Qp/Qs: relación flujo sanguíneo pulmonar y sistémico; Sat $\mathrm{O}_{2}$ : saturación de oxígeno.

Tabla 4. Recomendaciones para el cierre de ductus arterial permeable.

Recomendaciones para el cierre de ductus arterial permeable

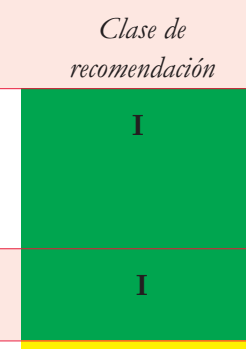

IIa

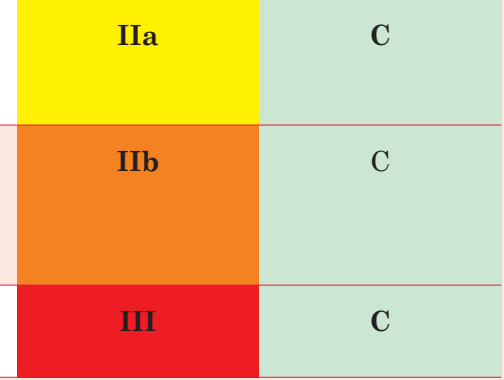

Se recomienda el cierre del DAP, independientemente de la presencia de síntomas, en pacientes con sobrecarga de volumen del VI sin evidencia de HTP (descartada mediante pruebas no invasivas o cateterismo cardíaco con $\mathrm{RVP}<3 \mathrm{UW})$.

Se recomienda el cierre en forma percutánea como técnica de elección, siempre y cuando sea técnicamente factible.

En pacientes que hayan desarrollado HTP con RVP entre 3 y 5 UW, debe considerarse el cierre cuando exista un shunt izquierda-derecha significativo $(\mathrm{Qp} / \mathrm{Qs}>1,5)$.

En pacientes que hayan desarrollado HTP con $R V P \geq 5$, podría considerarse el cierre en presencia de un shunt izquierda-derecha significativo (Qp/Qs $>1,5)$, luego de una evaluación individualizada realizada en centros de experiencia.

No se recomienda el cierre en pacientes con fisiología de Eisenmenger, HTP con $\mathrm{RVP} \geq 5$ o desaturación durante el ejercicio (Sat $\mathrm{O}_{2}<90 \%$ ).

DAP: ductus arterial permeable; VI: ventrículo izquierdo; HTP: hipertensión pulmonar; RVP: resistencias vasculares pulmona res; Qp/Qs: relación flujo pulmonar y sistémico; Sat $\mathrm{O}_{2}$ : saturación de oxígeno.

samente las dimensiones de la aorta en estos pacientes.

Los umbrales de severidad se adaptan de las Guías del 2017 para el manejo de las valvulopatías de la Sociedad Europea de Cardiología (ESC), considerando severas las estenosis aórticas con gra- diente medio $\geq 40 \mathrm{mmHg}$, velocidad máxima de 4 $\mathrm{m} / \mathrm{s}$; y área menor a $1 \mathrm{~cm}^{2}$, indexada de $0,6 \mathrm{~cm}^{2} / \mathrm{m}^{2} \mathrm{y}$ coeficiente de obstrucción de 0,25.

Está indicada la intervención en los pacientes sintomáticos con estenosis valvular severa clásica y de bajo flujo/bajo gradiente con fracción de eyec- 
ción del ventrículo izquierdo (FEVI) reducida, pero con reserva contráctil, así como en asintomáticos en los que se desenmascaran los síntomas con una prueba de ejercicio o cuya FEVI se encuentra disminuida en ausencia de otras causas.

Las estenosis aórticas supravalvulares pueden ocurrir como parte del síndrome de Williams-Beuren o aparecer de forma aislada, en general asociadas a una deleción o mutación del gen de la elastina. También se las han encontrado en el contexto de hipercolesterolemia familiar homocigótica. La mayoría de los pacientes sufren síntomas de obstrucción al tracto de salida del ventrículo izquierdo o isquemia miocárdica en la infancia. Es poco frecuente la presentación de la enfermedad en forma de muerte súbita. El tratamiento es primariamente quirúrgico y en general se puede considerar de forma más precoz que en los pacientes con estenosis valvular en el caso de que no requiera sustitución valvular aórtica. Se considera como umbral de indicación de intervención un gradiente medio $\geq 40 \mathrm{mmHg}$, y se puede considerar en pacientes con gradientes menores, pero con clínica claramente debida a la obstrucción (disnea de esfuerzo, angina o síncope) o en aquellos con FEVI disminuida en ausencia de otra causa. La tasa de éxito de la cirugía es alta. Puede hallarse insuficiencia aórtica hasta en un $25 \%$ de los pacientes, que no suele progresar tras la cirugía.

Las estenosis aórticas subvalvulares suelen estar relacionadas a otras anomalías congénitas como CIV, defectos del septo atrioventricular o como parte del complejo de Shone (acompañado de coartación aórtica, válvula mitral en paracaídas y estenosis supravalvular). En general se trata de un anillo o membrana fibrosa a nivel del tracto de salida del VI proximal a la válvula aórtica o un estrechamiento fibromuscular que debe ser correctamente diferenciado de la miocardiopatía hipertrófica obstructiva. Puede acompañarse de insuficiencia aórtica, por lo que es fundamental diagnosticarla y estadificarla. La cirugía es el método correctivo de elección y suele tener tasas de éxito altas. Puede ocurrir reestenosis, lo que requiere seguimiento por un equipo especialista en CC del adulto.

Las indicaciones de intervención están dadas por un gradiente medio $\geq 40 \mathrm{mmHg}$ en pacientes sintomáticos, así como en pacientes con gradientes menores, pero con insuficiencia valvular severa, disfunción del VI, hipertrofia severa, etcétera.

\section{Coartación de aorta}

La coartación de aorta (CoA) es definida como una arteriopatía generalizada más que como una disminución puntual del calibre de la aorta. Su espec- tro de presentación comprende desde estrechamientos localizados hasta largos segmentos involucrados con arco aórtico hipoplásico. Su característica principal es una constricción a nivel de la aorta torácica distal a la desembocadura del ductus. Otras localizaciones son extremadamente raras.

Suele existir asociación lesional muy frecuente con VAB en hasta un $85 \%$ de los casos, y en menor medida CIV, DAP, estenosis valvular aórtica o estenosis subaórtica, defectos mitrales como el complejo de Shone y válvula mitral en paracaídas, entre otros. Dentro de los defectos extracardíacos predominan los aneurismas intracraneales, circulación colateral y nacimiento anómalo de la subclavia derecha (subclavia derecha ilusoria). Los pacientes con síndrome de Turner tienen mayor riesgo de padecer CoA.

Presentación clínica. La severidad de la $\mathrm{CoA}$ suele relacionarse con la manifestación clínica y con la edad de presentación. Los pacientes con CoA severas manifestarán síntomas a edades tempranas, mientras que los estrechamientos más leves pueden cursar asintomáticos hasta la vida adulta, alcanzando el diagnóstico con la búsqueda etiológica de hipertensión arterial (HTA). Los síntomas típicos incluyen disnea, dolor torácico, frialdad y/o claudicación de miembros inferiores con pulsos débiles o ausentes, cefaleas, epistaxis, tinnitus, etcétera.

Los pacientes que alcanzan la adolescencia suelen tener adecuadas tasas de supervivencia hasta la sexta década. La morbilidad a largo plazo es común y suele estar vinculada a enfermedades de la aorta e HTA. Se trata de una patología que puede complicarse con infarto de miocardio o accidente cerebrovascular precoz por ateromatosis acelerada, endocarditis infecciosa, hemorragias intracraneales, insuficiencia cardíaca, disección y rotura aórtica.

Evaluación diagnóstica. En el diagnóstico de CoA, se considera significativa la presencia de un gradiente de presión (toma no invasiva PA) $\geq 20$ $\mathrm{mmHg}$ entre extremidades superiores e inferiores, así como la atenuación o ausencia de pulsos en extremidades inferiores con retardo del pulso radio femoral. Estos pacientes suelen acompañarse de HTA (en miembros superiores), auscultación anormal con soplo sistólico interescapular o frémito precordial a causa de la circulación colateral. El característico "signo del tres" se observa en la radiografía de tórax por desgaste costal en casos donde la circulación colateral es extensa. La ecocardiografía transtorácica (ETT) es el primer estudio de elección ante la sospecha de CoA que valora la extensión y severidad anatómica de la CoA. Aporta 
Tabla 5. Recomendaciones de intervención en la coartación de aorta y recoartación.

\begin{tabular}{lcc}
\hline Recomendaciones para la intervención en coartación y recoartación de aorta & $\begin{array}{c}\text { Clase de } \\
\text { recomendación }\end{array}$ & Nivel de evidencia \\
\hline $\begin{array}{l}\text { Se recomienda la reparación de CoA o reCoA en pacientes hipertensos a } \\
\text { con gradiente de presión } \geq 20 \mathrm{mmHg} \text { entre extremidades superiores e } \\
\text { inferiores confirmado en forma invasiva, con preferencia por el } \\
\text { cateterismo sobre la cirugía para el tratamiento cuando sea técnicamente } \\
\text { factible. }\end{array}$ & I & C \\
\hline $\begin{array}{l}\text { Se debe considerar el tratamiento mediante cateterismo (stent) en } \\
\text { pacientes hipertensos con estrechamiento } \geq 50 \% \text { entre el segmento } \\
\text { coartado y la aorta descendente a nivel del diafragma, aun si el gradiente } \\
\text { invasivo es <20 mmHg. }\end{array}$ & IIa & C \\
\hline $\begin{array}{l}\text { Se debe considerar la reparación de CoA o reCoA preferiblemente } \\
\text { mediante cateterismo, en pacientes normotensos con gradiente de presión } \\
\geq 20 \text { mmHg entre extremidades superiores e inferiores confirmado en }\end{array}$ & IIa \\
$\begin{array}{l}\text { forma invasiva (gradiente trans-CoA), cuando sea técnicamente factible. } \\
\begin{array}{l}\text { Se puede considerar el tratamiento mediante cateterismo (stent) en } \\
\text { pacientes normotensos con estrechamiento } \geq 50 \% \text { entre el segmento } \\
\text { coartado y la aorta descendente a nivel del diafragma, aun si el gradiente } \\
\text { invasivo es <20 mmHg. }\end{array}\end{array}$ \\
$\begin{array}{l}\text { CoA: coartación; ReCoA: recoartación; a: se recomienda la toma de presión en el brazo derecho. } \\
\text { IIb }\end{array}$ \\
\hline
\end{tabular}

datos sobre la función sistólica, grosor parietal del ventrículo izquierdo y anomalías asociadas. En la actualidad no se considera confiable la valoración de severidad mediante gradientes trans-CoA con Doppler, ya que puede estar subestimado como en casos de extensa circulación colateral o disfunción ventricular o sobrestimado por la presencia de stent o implante de conductos quirúrgicos que determinan menor complacencia aórtica. En estos casos, tanto el arrastre diastólico como la ausencia de pulsatilidad de la aorta abdominal son dos parámetros que indican CoA significativa.

La resonancia magnética $(\mathrm{RM})$ y la angiotomografía (angio TC) preferiblemente con reconstrucción 3D se imponen por su utilidad para diagnóstico y seguimiento. La evidencia imagenológica de un estrechamiento mayor al 50\% del diámetro aórtico a nivel del diafragma también es criterio para intervención.

La medición invasiva del gradiente pico-pico trans-CoA se realiza en el curso de una angioplastia con stent o con balón. Se considera significativo un valor $\geq 20 \mathrm{mmHg}$.

Manejo terapéutico. La estrategia de elección para la población adulta es la angioplastia con implante de stent vía percutánea. La angioplastia con balón se reserva para redilatación de stents previamente implantados. La estrategia quirúrgica se reserva para casos no factibles para tratamientos percutáneos. El uso de stents cubiertos es de elección por sus menores tasas de complicaciones a corto y largo plazo, aunque no se recomiendan en pacientes que no han alcanzado el tamaño adulto ante el requerimiento de redilataciones futuras.

Dado lo sistémico de esta patología con afectación vascular parietal difusa, se deberán tomar en consideración otras potenciales intervenciones estructurales, como es el caso de estenosis o insuficiencia valvular aórtica significativas o dilatación aneurismática de aorta ascendente (en caso de BAV), aneurismas o pseudoaneurismas en sitios de reparación previa o aneurismas del polígono de Willis. Las recomendaciones de intervención se resumen en la tabla 5 .

Seguimiento y recomendaciones. Tanto la HTA en reposo como la hallada durante el ejercicio son frecuentes en pacientes con CoA, inclusive luego de una reparación exitosa, y representa un importante factor de riesgo para enfermedad coronaria prematura, disfunción ventricular y cuadros catastróficos como ruptura aórtica o de aneurismas cerebrales. Se menciona a la forma "gótica" del arco aórtico y segmentos hipoplásicos como responsables de su desarrollo.

Se recomienda un seguimiento frecuente y la toma de presión arterial (PA) en reposo en ambos miembros, así como en forma ambulatoria. Sin embargo, el significado de una respuesta hipertensiva aislada durante pruebas de estrés con ejercicio está menos establecida. Se exhorta seguir las directrices 2018 ESC/ESH para el manejo de HTA.

La recoartación es una complicación frecuente y puede inducir o agravar la HTA y sus respectivas complicaciones. Se establece bajo los mismos parámetros que para el diagnóstico de CoA. Su hallazgo 
requiere la realización de un cateterismo para su confirmación y tratamiento.

Otras causas de reintervenciones en estos pacientes son la existencia de aneurismas o pseudoaneurismas en la zona de reparación, con mayor riesgo de ruptura parietal. La reparación quirúrgica con implante de parche presenta puntualmente mayor riesgo de formación de aneurismas, mientras que los conductos favorecen la formación de pseudoaneurismas.

Dado el carácter sistémico y crónico de esta patología, se exhorta a un seguimiento anual de todos los pacientes y la realización periódica (de 3 a 5 años) de estudios de imagen avanzada (preferiblemente RM) antes y después de una intervención para descartar las complicaciones antedichas.

Los pacientes reparados con éxito, sin gradiente residual y sin HTA en reposo o ejercicio pueden realizar ejercicio sin restricciones. Sin embargo, los pacientes con gradiente residual, HTA o complicaciones de la pared deben evitar el ejercicio isométrico.

El embarazo suele ser bien tolerado en mujeres con reparación exitosa. Sin embargo, aquellas con CoA no reparada o gradientes residuales significativos, HTA o complicaciones de la pared suelen tener mayor riesgo de ruptura aórtica o de aneurismas cerebrales.

Se recomienda la profilaxis antibiótica para endocarditis infecciosa en los pacientes de alto riesgo.

\section{Lesiones obstructivas del corazón derecho}

Las obstrucciones del tracto de salida del ventrículo derecho pueden ser subinfundibular, infundibular, valvular o supravalvular.

La estenosis infundibular suele ocurrir con otras lesiones como la CIV, la tetralogía de Fallot y la estenosis valvular pulmonar.

La estenosis valvular pulmonar puede ser una lesión aislada y acompañarse de dilatación del tronco pulmonar y/o de las arterias pulmonares. Comúnmente las válvulas tienen una típica forma de cúpula con una abertura central estrecha, pero con movimiento de su base conservado. Las válvulas displásicas son considerablemente menos frecuentes.

La estenosis pulmonar supravalvular o de las arterias pulmonares suele ocurrir en el contexto de un síndrome o malformaciones asociadas como tetralogía de Fallot, síndrome de Williams-Beuren, síndrome de Noonan, síndrome de Alagille, entre otros. La estenosis puede localizarse a nivel del tronco, la bifurcación o las ramas pulmonares, puede ser discreta o difusa (a lo cual se denomina comúnmente "hipoplasia") u oclusión total. Pueden ocurrir en el sitio de colocación de un cortocircuito previo o de inserción de un parche o tubo en dicho sector. Las estenosis de entidad considerable (mayores al 50\% de la luz del vaso) suelen generar gradiente de presión y, por lo tanto, HTP en el sector proximal.

El ETT es la técnica de primera línea para valorar las obstrucciones del tracto de salida del VD. La correlación entre velocidades de flujo y gradientes solo puede tomarse como fidedigna si las estenosis son puntuales. En presencia de función normal del VD se considera que la obstrucción es severa si el gradiente supera los $64 \mathrm{mmHg}$, y leve si es menor de $36 \mathrm{mmHg}$. Cabe destacar que, si la estenosis es de longitud considerable, o existen varias lesiones en tándem, la ecuación de Bernoulli puede sobrestimar el gradiente de presión por lo que debería utilizarse la velocidad del flujo de insuficiencia tricúspide para valorar la presión en el VD y, por ende, la gravedad de la obstrucción al tracto de salida.

Se recomienda la valvulotomía percutánea con balón para los pacientes con estenosis pulmonar valvular en válvulas no displásicas, o en estenosis supravalvulares pulmonares (si es técnicamente posible). En estas últimas se suele requerir colocación de stent. La cirugía se reserva para las estenosis severas subinfundibulares o infundibulares con anillo hipoplásico, en válvulas displásicas o en aquellos que tengan lesiones asociadas como insuficiencia pulmonar o tricúspide severas.

Los pacientes con este tipo de patologías requieren de seguimiento ecográfico y clínico durante toda su vida, con una asiduidad dependiente de la gravedad de la obstrucción y de la técnica de reparación.

\section{Anomalía de Ebstein}

La anomalía de Ebstein se caracteriza por el desplazamiento apical de las valvas de la tricúspide, lo que genera una atrialización de parte del VD. La valva anterior suele originarse a nivel anular, pero se encuentra elongada, mientras que las valvas septal y posterior están desplazadas hacia el ápex y a menudo insertas en el endocardio. En general, esta válvula malformada muestra grados de insuficiencia variable. Por sus características se asocia a otras anomalías congénitas como múltiples vías accesorias, CIA tipo ostium secundum, entre otras.

La gravedad desde el punto de vista hemodinámico varía según el grado de insuficiencia, la entidad del VD funcional y la presencia de cortocircuitos entre cámaras derechas e izquierdas. 
Tabla 6. Indicaciones de intervención en la insuficiencia pulmonar posterior a la corrección de la TdF.

\begin{tabular}{|c|c|c|}
\hline $\begin{array}{l}\text { Recomendaciones para la intervención en la insuficiencia pulmonar posterior } \\
\text { a la reparación de la } T d F\end{array}$ & $\begin{array}{l}\text { Clase de } \\
\text { recomendación }\end{array}$ & Nivel de evidencia \\
\hline $\begin{array}{l}\text { Se recomienda el reemplazo valvular pulmonar en pacientes sintomáticos } \\
\text { con IP severa y/u obstrucción al tracto de salida del VD al menos } \\
\text { moderada }\end{array}$ & I & C \\
\hline $\begin{array}{l}\text { En pacientes sin tractos de salida nativos }{ }^{b} \text {, se prefiere la intervención } \\
\text { percutánea con catéter si es anatómicamente posible. }\end{array}$ & $\mathbf{I}$ & C \\
\hline $\begin{array}{l}\text { El reemplazo valvular pulmonar debe ser considerado en pacientes } \\
\text { asintomáticos con IP severa y/u obstrucción al tracto de salida del VD } \\
\text { cuando al menos uno de los siguientes criterios está presente: } \\
\text { - descenso objetivo de la capacidad funcional; } \\
\text { - dilatación progresiva del VD a valores de volumen sistólico final } \\
\geq 80 \mathrm{~mL} / \mathrm{m}^{2} \text {, y/o diastólico final } \geq 160 \mathrm{~mL} / \mathrm{m}^{2 c} \text {, y/o progresión de la } \\
\text { insuficiencia tricúspide al menos moderada; } \\
\text { - progresión de la disfunción sistólica del VD; } \\
\text { - obstrucción al tracto de salida del VD con presión sistólica del VD } \\
>80 \mathrm{mmHg} \text {. }\end{array}$ & IIa & C \\
\hline
\end{tabular}

Es frecuente que estos pacientes se presenten clínicamente por taquiarritmias supraventriculares (por lo general, taquicardia por rentrada aurículo-ventricular dependiente de vía accesoria), y el examen de primera línea para el diagnóstico y valoración de la severidad es el ETT, si bien después puede completarse con un enfoque desde la multimodalidad de imagen.

En cuanto al tratamiento de estos pacientes, se encuentra guiado por lo síntomas. Los trastornos del ritmo sintomáticos pueden manejarse médicamente o, preferentemente, mediante ablación por radiofrecuencia. En cuanto a la corrección quirúrgica, es fundamental que sea realizada por cirujanos cardíacos especializados en CC, y está indicado en aquellos pacientes con insuficiencia de la válvula severa sintomática; o por síntomas desencadenados tras prueba de esfuerzo.

\section{Tetralogía de Fallot}

La tetralogía de Fallot (TdF) se produce como consecuencia de una desviación anterógrada del septum infundibular que determina cuatro componentes que constituyen la patología: CIV no restrictiva, cabalgamiento aórtico menor al 50\%, obstrucción al tracto de salida del VD (que puede ocurrir a cualquier nivel) e hipertrofia ventricular derecha.

La corrección quirúrgica suele suponer el cierre de la CIV, corrección de la obstrucción al tracto de salida y si corresponde, valvulotomía pulmonar. Muchos lo logran utilizando un parche transanular entre el VD y la arteria pulmonar. A continua- ción se citan las complicaciones más frecuentemente encontradas en el adulto con TdF reparada.

\section{Insuficiencia pulmonar (IP)}

Algún grado de IP es prácticamente la norma en la edad adulta tras la reparación de TdF. Si esta es severa, con el tiempo suele generar dilatación y disfunción sintomática del VD. La gravedad de la IP puede aumentar si el paciente presenta, además, estenosis en el tronco o arterias pulmonares y/o HTP.

La IP severa es la causa más frecuente que motiva la cirugía en los pacientes adultos corregidos. Sus indicaciones pueden verse resumidas en la tabla 6 . Sin embargo, debe recordarse la alternativa del implante percutáneo de válvula protésica a nivel del tracto de salida del VD, que también puede considerarse en los pacientes con obstrucción.

Cabe destacar que el método de elección para la cuantificación del volumen y función del VD, y por lo tanto necesario para la toma de decisiones terapéuticas, es la RM.

\section{Obstrucción al tracto de salida del VD residual}

Puede ocurrir a nivel infundibular, valvular pulmonar, tronco pulmonar o de ramas.

\section{Arritmias auriculares, ventriculares y muerte súbita cardíaca}

Las complicaciones arrítmicas son, por lo general, tardías y de gran entidad. La prevalencia de arritmias auriculares en los pacientes reparados a lo largo de su vida es del 20\%. Suelen involucrar al 
Tabla 7. Indicaciones de intervención posterior a la cirugía de switch arterial en la TGA.

\begin{tabular}{lcc}
\hline $\begin{array}{l}\text { Recomendaciones para la intervención en pacientes con cirugía de switch arterial por } \\
\text { transposición de grandes arterias }\end{array}$ & $\begin{array}{c}\text { Clase de } \\
\text { recomendación }\end{array}$ & Nivel de evidencia \\
\hline $\begin{array}{l}\text { La intervención quirúrgica o percutánea con implante de stent (según el } \\
\text { sustrato) está recomendada para las estenosis coronarias que causan } \\
\text { isquemia. }\end{array}$ & I \\
\hline $\begin{array}{l}\text { La cirugía sobre la raíz de la neoaorta debe ser considerada cuando esta } \\
\text { tiene un diámetro mayor a } 55 \text { mm. }\end{array}$ & IIa \\
\hline $\begin{array}{l}\text { La colocación de stent sobre estenosis de ramas pulmonares debe ser } \\
\text { considerada independientemente de los síntomas, si ésta es mayor al } 50 \% \\
\text { del diámetro y la presión sistólica en el VD es mayor a } 50 \text { mmHg y/o se } \\
\text { objetiva disminución de la perfusión en el pulmón afectado. }\end{array}$ & IIa & C \\
\hline $\begin{array}{l}\text { VD: ventrículo derecho. } \\
\text { C }\end{array}$ & \\
\hline
\end{tabular}

istmo cavotricuspídeo y a la incisión quirúrgica sobre la aurícula derecha.

Cuando suceden las arritmias ventriculares (taquicardia o fibrilación ventricular), es importante reconocerlas, más aún si el paciente presenta disfunción ventricular. La frecuencia de muerte súbita cardíaca en estudios retrospectivos es de 1\%-3,5\% en este grupo de pacientes, por lo que la correcta estratificación del riesgo y selección de los pacientes candidatos a terapias de prevención es fundamental.

Los factores de riesgo asociados a la ocurrencia de arritmia ventricular y muerte súbita son: la duración del QRS mayor a 180 ms, disfunción ventricular izquierda o derecha y taquicardia ventricular inducible por estudio electrofisiológico. Es importante destacar que, cuando es posible, la ablación del sustrato generador de dichas arritmias ventriculares suele ser muy eficaz.

El implante de cardiodesfibrilador (DAI) está indicado como prevención secundaria de la muerte súbita (indicación clase IC). Sin embargo, el implante como prevención primaria sigue siendo tema de discusión y aún no se ha desarrollado una posición sobre este. De todas formas, los pacientes considerados de alto riesgo de muerte súbita arrítmica deben ser considerados para el implante de DAI. Las recomendaciones de intervención se resumen en la tabla 6 .

\section{Transposición de grandes arterias}

La transposición de grandes arterias (TGA) se define como aquella que presenta concordancia aurículo-ventricular y discordancia ventrículo-arterial. La TGA simple es aquella que no se acompaña de defectos congénitos adicionales (de presentarlos, se denomina complejo). Existe también la TGA corregida de manera congénita, en la cual se produce una doble discordancia (aurículo-ventricular y ventrículo-arterial).
Las complicaciones tras la cirugía de switch arterial por TGA simple son: dilatación de la raíz neoaórtica que produce insuficiencia aórtica; estenosis pulmonar supravalvular y estenosis de rama pulmonar (uni o bilateral) que se produce como consecuencia de la posición de bifurcación pulmonar anterior a la aorta ascendente tras técnica de Lecompte; disfunción ventricular izquierda y arritmias ventriculares; isquemia por complicaciones tras reimplante de arterias coronarias, obstrucción al tracto de salida del VI (por angulación excesiva, muy poco frecuente), entre otras.

La supervivencia a los 30 años del procedimiento es muy alta, mayor al $90 \%$, y de $60 \%$ a $80 \%$ en aquellos supervivientes con ausencia de complicaciones.

Los estudios complementarios para el diagnóstico de complicaciones son el ecocardiograma en reposo y estrés (para evaluar isquemia inducible), la RM para valoración cuantitativa más confiable, la angio TC coronaria, las técnicas de imagen nuclear como el centellograma de perfusión pulmonar, si bien no se utiliza de primera línea, puede cobrar importancia cuando las otras técnicas no están disponibles o haya resultados no concluyentes.

Las indicaciones de intervención tras la operación de switch arterial pueden observarse en la tabla 7 .

\section{Conclusiones}

Hemos recorrido las diferentes lesiones cardíacas congénitas y su presentación en adultos, resumiendo los aspectos clínicos diagnósticos y terapéuticos considerados más importantes. De esto se desprende la necesidad de contar con un equipo especializado en el manejo del paciente adulto con $\mathrm{CC}$ en todos sus niveles, enfatizando la singularidad y dificultad del manejo. 
Guillermo Agorrody, ORCID: 0000-0002-9241-6681

Lorena Dieguez, ORCID: 0000-0002-0206-1099

Este artículo fue aceptado para su publicación por la Dra. Victoria Ramos

\section{Bibliografía}

1. Baumgartner H, De Backer J, Babu-Narayan SV, Budts W, Chessa M, Diller GP, et al. 2020 ESC Guidelines for the management of adult congenital heart disease: the task force for the management of adult congenital heart disease of the European Society of Cardiology (ESC). Endorsed by: Association for European Paediatric and Congenital Cardiology (AEPC), International Society for Adult Congenital Heart Disease (ISACHD). Eur Heart J. 2021;42(6): 563-645. DOI: 10.1093/eurheartj/ehaa554. 